\title{
System Biology: An Emerging Toolkit for Improved Tyrosine Kinase Inhibition Therapy for Myeloproliferative Neoplasms
}

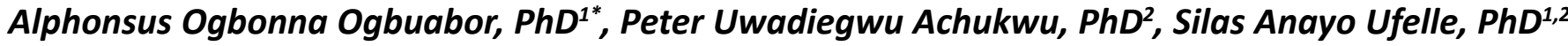 and Daniel Chukwuemeka Ogbuabor, PhD ${ }^{1,3}$}

\author{
${ }^{1}$ Department of Hematology, Enugu State University of Science and Technology Teaching Hospital, Parklane, \\ Enugu, Nigeria \\ ${ }^{2}$ Department of Medical Laboratory Sciences, Faculty of Health Sciences and Technology, College of Medicine, \\ University of Nigeria, Enugu, Nigeria
}

${ }^{3}$ Department of Health Administration and Management, Faculty of Health Sciences and Technology, University of Nigeria, Enugu, Nigeria

*Corresponding author: Ogbuabor Alphonsus Ogbonna, PhD, Department of Hematology, Enugu State University of Science and Technology Teaching Hospital, Parklane, Enugu, Nigeria, Tel: +234-07032753784

\begin{abstract}
Concerted efforts have been made by scientists over the last decades in elucidating the molecular mechanisms leading to myeloproliferative neoplasms. The identification of oncogenic mutations in signal transduction pathways revealed the role of specific pathways in inducing excessive proliferation of myeloid lineages. The subsequent development of mouse models carrying mutations found in patients proved that the aberrant activation of these specific pathways plays a crucial role in the pathology of myeloproliferative neoplasms. This led to the development of tyrosine kinase inhibitors for the treatment of patients. However, reduced selectivity, incomplete inhibition, drug resistance and drug induced toxicities remains a major challenge with current therapy regimens. System biology is a novel technology that holds great potentials for achieving better clinical outcomes for tyrosine kinase inhibition therapy. This review presents an overview on the need to apply system biology techniques in developing improved tyrosine kinase inhibitors for myeloproliferative neoplasms.
\end{abstract}

\section{Keywords}

Myeloproliferative neoplasms, Tyrosine kinase inhibitors, Therapy, System biology

\section{Introduction}

Myeloproliferative neoplasms are a heterogeneous group of hematological malignancies characterized by an increased clonal proliferation of one or more myeloid lineages [1,2]. According to the World Health Organization (WHO), the classical myeloproliferative neoplasm can be divided into two major groups namely the Philadelphia chromosome positive group which includes chronic myeloid leukemia (CML) and the Philadelphia chromosome negative groups which includes Essential Thrombocythemia (ET), Polycythemia Vera (PV) and Primary Myelofibrosis (PMF) [3]. Due to an underlying clonal proliferation, patients with myeloproliferative neoplasms suffer complications including thrombosis and haemorrhage. Over time, patients develop progressive bone marrow failure and may also transform to acute myeloid leukemia. Studies identified three main mechanisms of pathophysiology which include (i) Somatic driver mutations that stimulate activation of various tyrosine kinase pathways, (ii) Cooperating driver mutations in myeloid genes and, (iii) Uncommon genetic factors that initiate different clinical neoplastic phenotypes [4].

\section{Oncogenes in Myeloproliferative Neoplasms}

Underlying myeloproliferative neoplasms are abnormalities in various genes involved the tyrosine kinase signaling pathways [5]. These may include:

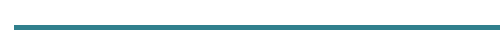

Citation: Ogbuabor AO, Achukwu PU, Ufelle SA, Ogbuabor DC (2020) System Biology: An Emerging Toolkit for Improved Tyrosine Kinase Inhibition Therapy for Myeloproliferative Neoplasms. Int J Blood Res Disord 7:060. doi.org/10.23937/2469-5696/1410060

Accepted: November 14, 2020: Published: November 16, 2020

CLINME D

INTERNATIONAL LIBRARY

Copyright: (C) 2020 Ogbuabor AO, et al. This is an open-access article distributed under the terms of the Creative Commons Attribution License, which permits unrestricted use, distribution, and reproduction in any medium, provided the original author and source are credited.
} 


\section{ABL mutations}

Abelson murine leukemia viral gene (ABL) located on chromosome 9q34.1 is a cytoplasmic tyrosine kinase. Physiological functions of $A B L$ include nonerythoid myelopoiesis and cytoskeletal rearrangement including regulation of small GTPases, inhibition of cell migration and F-actin binding. The most extensively studied $A B L$ mutation is BCR-ABL; the disease causing mutation in chronic myeloid leukemias [6].

\section{JAK 2 mutations}

JAK 2 is a cytoplasmic tyrosine kinase required for signal transduction from type 1 cytokine receptors which include those for thrombopoientin, erythropoientin and granulocyte colony stimulating factor and therefore play a vital role in myelopoiesis. A valine to phenylalanine substitution at codon 617 (V617F) of the JAK2 gene has been identified in over $95 \%$ of patients with polycythemia vera, $50-60 \%$ of those with essential thrombocythemia or primary myelofibrosis. JAK2 exon 12 mutations has also been identified in small proportion of JAK2V617F negative polycythemia vera patients with a frequency of 2-3\% [7].

\section{MPL mutations}

MPL is located in chromosome 1p34 and encodes for the thrombopoientin receptor. A missense mutation in the exon 10 of the myeloproliferative leukaemia (MPL), the human homologue of the murine myeloproliferative leukaemia virus oncogene which is the cell surface receptor for thrombopoientin such as $\mathrm{M} 515 \mathrm{~L}$ and M515K(less commonly S505N) have been reported in patients with ET and MF.MPL mutations are associated with increased JAK-STAT,ERK and AKT signaling [7].

\section{CALR mutations}

Calreticulin (CARL) is known to be involved in the regulation of calcium uptake and release in endoplasmic reticulum and acts as a chaperone together with calnexin and ERp57 to form part of the regulatory machinery involved in the folding and quality control of newly synthesized glycoproteins. Mutations in the calreticulin gene are also found in approximately $25-35 \%$ of patients with ET and $35-40 \%$ of those with MF [8].

\section{LNK mutations}

The lymphocyte specific adaptor protein also known as Src homology 2B3 (SH2B3) is an adaptor protein that negatively affects the JAK-STAT signaling. Loss of function mutations of LNK at exon 2 have been reported in myeloproliferative patients [2].

\section{CBL mutations}

Casitas B-lineage lymphoma proto-oncogene is an E3 ubiquitin ligase which is specifically involved in the ubiquitination of a number of receptor tyrosine kinases as well as having a role in intracellular signal transduction. Its targets include PDGFR, c-KIT, FLT3 and MPL mutations in the RING domain responsible for CBL's ligase activity have been described in myeloproliferative neoplasms [7].

\section{Other mutations}

A number of other mutations including the SOCS protein another class of regulator of JAK2 signaling and mutations increasing the RAS pathway signaling have also been observed in a small number of patients. Recently, some developmental pathways such as MAPK, Hedgehog, Wnt and Notch have been shown to play a role and might be a promising area of research as possible therapeutic targets.

\section{Tyrosine Kinase Inhibition Therapy}

Advances in the understanding of the oncogenic activation of tyrosine kinases in myeloproliferative neoplasms has led to the development of novel tyrosine kinase inhibition therapy such as BCR-ABL + Inhibitors, P13K inhibitors and JAK2 inhibitors [9].

\section{BCR-ABL + Inhibitors}

The discovery of BCR-ABL was one of the most influential findings for the treatment of myeloproliferative neoplasms as this eventually identified the first target for specific tyrosine kinases, paving the way for direct drug therapies in patients [10]. Imatinib (STI 571) was developed as the first molecular targeted therapy that specifically inhibits the BCR-ABL tyrosine kinase activity in patients with chronic myeloid leukemia. Due to its excellent hematologic responses in patients, Imatinib was approved as the first line treatment for newly diagnosed CML [10]. However, resistance to the drug has been frequently reported and is attributed to the fact that transformation of hematopoietic stem cells by $B C R-A B L$ is associated with genomic instability with point mutations within the $A B L$ tyrosine kinase of the $B C R-A B L$ oncoprotein identified as the major cause of resistance though over expression of the BCR-ABL protein and some acquired cytogenetic aberrations have also been reported [11]. A variety of strategies including the escalation of Imatinib, combination of Imatinib with chemotherapeutic drugs, alternative $B C R-A B L$ inhibitors and inhibitors of kinases acting downstream of BCR-ABL lead to the design of newer $B C R-A B L$ inhibitors such as AMN107, BMS354825, ONO12380 yet it remains a challenge to creating a new drug that are more potent than Imatinib and/or more effective against Imatinib resistant BCR-ABL clones [11].

\section{P13K pathway inhibitors}

The phosphatidylinositol 3-kinase enzyme is involved in the control of protein synthesis, growth, survival and proliferation of myeloid cells. The P13K pathway plays a central role in the pathogenesis of myeloproliferative neoplasms. Its molecular signaling has emerged as one 
of the most attractive targets in myeloproliferative neoplasms due to its central role in integrating signals from different receptor kinases such as AKT and mTOR to regulate cell proliferation. The P13K pathway inhibitors can be broadly classified as inhibitors of P13K, AKT and mTOR. Though some of these molecules hold promise, the success so far has been limited. Clinical trials with GSK1059615, a P13K inhibitor, GSK690693 an AKT inhibitor and AZD2014 an mTOR inhibitor has been suspended due to drug induced toxicities [8]. Newer inhibitors which can target $\mathrm{P} 13 \mathrm{~K}$ pathway at more than one node commonly referred to as dual-specificity inhibitors and P13K inhibitors in combination with other chemotherapeutic agents has shown limited efficacy [8].

\section{JAK2 inhibitors}

Following the identification of activating mutations in the JAK-STAT pathway, there were concerted efforts put into the development of substances that can target the kinase activity of JAK2. These are commonly referred to as the JAK inhibitors. JAK inhibitors could be classified into two groups of allosteric and non-allosteric inhibitors depending on their mechanism of action and region targeted for therapy.

\section{Non-allosteric inhibitors}

There are two types namely the type 1 inhibitors and type II inhibitors. While type I inhibitors target the ATP-binding site of JAK2 in the active conformation of the kinase domain, the type II inhibitors target the ATP-binding site of its kinase domain in the inactive conformation. Type I inhibitors have been prominent in the management of myeloproliferative disorders [12]. Ruxolitinib, a Type I inhibitor has been recently approved for the treatment of myeloproliferative disorders by the US food and Drug Administration. Though, it is still at the early stages of clinical trials. Type II inhibitors are more effective and powerful than type I inhibitors. Two type II inhibitors (NVP - BBT594 and NVP-CH2868) have been developed. The NVP - BBT594 was effective in myeloproliferative neoplasm cellular models while NVP$\mathrm{CH} 2868$ has been effective in preclinical mouse myeloproliferative models $[12,13]$.

\section{Allosteric inhibitors}

These are inhibitors which bind to other sites different from the ATP-kinase binding active sites. There are two types namely type III inhibitors which bind to a site close to the ATP-binding site (e.g. LS104) and type IV inhibitors which bind to an allosteric site far from the ATP-binding Site (e.g. ONO44580). Targeting regions outside the ATP-binding site provides an advantage of higher selectivity and the possibility to overcome drug resistance by targeting two different sites in a combinatorial therapy model $[14,15]$. There are currently no JAK allosteric inhibitors in clinical use.

\section{The challenges of tyrosine kinase inhibition ther- apy}

The aim of tyrosine kinase inhibition therapy for myeloproliferative neoplasms has not been achieved. There are limitations with respect to limited efficacy, selectivity and dose-limited toxicities [16]. A major mechanism of this is the acquisition of secondary mutations in the protein being targeted. This has been shown in the Abl-kinase inhibitors for chronic myeloid leukemia [17]. A second mechanism is the activation of alternate, bypass pathways in some malignancies. In EGFR mutant non-small cell lung cancer treatment, EGFR inhibitors can lead to downstream activation of P13K signaling in an EGFR - dependent manner. Such a mechanism is possible in myeloproliferative neoplasms as well [18]. A third mechanism is insufficient pathway inhibition at clinically tolerable doses. JAK2 inhibitors in clinical development are not specific for mutant JAK2 and can also efficiently inhibit wild type JAK2. Therefore, using doses that are capable of inhibiting mutant JAK2 activity is bound to also have adverse effects on normal hematopoiesis. This is the cause of the dose limiting toxicities involving profound cytopenias observed in treatment with JAK2 inhibitors [19]. There is therefore need for new inhibitors or combination therapies which will not only ameliorate symptoms but could arrest clonal proliferation of the myeloid stem cells. New generation of tyrosine kinase inhibitors targeting unique sequences for the signaling pathways need to be developed. Such inhibitors will be less toxic and will target the clonal complexities of the disease [20].

\section{Perspective for a system biology framework}

System biology provides a network analysis of disease related genes and drug targeting of signaling pathways which could enable the discovery of drugs with the potential desired effects for a given disease [20-22]. It enables integrated view of biological systems by studying all the translated proteins in a cell [23]. This can be applied to enhance our understanding of the complexity of multiple oncogenic mechanisms implicated in myeloproliferative neoplasms with respect to tyrosine kinase inhibitor interactions. Integrated computational technologies are currently being explored for various cancer phenotypes to identify common mechanisms that drive the clonal proliferation of cells including aberrant metabolisms and possible tolerant drug doses or combination therapies [24]. In a recent study, Shank, et al. [16] designed a mathematical model consisting of multiple components that was parameterized using in vitro data which predicted the efficacies of different dosing strategies for Ruxolitinib in silico enabling the testing of the hypothesis about the best intermittent treatment strategies to be validated in an in vivo myeloproliferative murine model. Through this, it was shown that an intermittent treatment strategy could lead to increased efficacy and/or reduced toxicity in patients. 


\section{Experimental design for a system biology inter- vention}

Since signaling through the tyrosine kinase pathways are involved in a plethora of myeloproliferative neoplasms, an experimental intervention may begin by sketching all the protein interactions that are involved in the cellular responses for the pathways during haematopoiesis. After determining the protein interactions, mass action kinetics is applied to describe the speed of protein interactions in the system. Each protein interaction could be described by a Michealis Menten reaction. Through this a differential equation to model the protein interactions as a mathematical model for the selectivity of drug regimens for specific types of myeloproliferative neoplastic disorders in which experiments can determine the parameter values could be developed using a deterministic or stochastic model. The parameter values gives the reaction rates of each protein interaction in the system $[25,26]$. Through this, the protein behaviors for particular neoplastic processes could be determined thus providing insights into the specific drug interactions with individual proteins [27]. Unknown reaction rates are determined by simulating the model of known parameters and their target behaviors which provides possible parameter values for cellular response.

\section{Conclusion}

Tyrosine kinase inhibition remains a novel therapeutic target for myeloproliferative neoplasms. System biology presents a dynamic tool that could be strategically applied in tyrosine kinase inhibition for designing dose regimens, drug repurposing and combination therapies for future interventions on patients with myeloproliferative neoplasms. Its application will particularly enable the development of mutant-specific tyrosine kinase inhibitors that can spare normal cells but target proliferative and pro-survival pathways which are activated thereby eliminating drug toxicities.

\section{References}

1. Pinilla-lbarz J, Sweet KL, Corrales-Yepez GM, Komrokji RS (2016) Role of tyrosine-kinase inhibitors in myeloproliferative neoplasms: comparative lessons learned. Onco Targets Ther 9: 4937-4957.

2. Sonbol MB, Firwana B, ZarzourA, Morad M, Rana V, et al. (2013) Comprehensive review of JAK inhibitors in myeloproliferative neoplasms. Ther Adv Hematol 4: 15-35.

3. Koopmans SM, Marion AMW, Schouten HC (2012) Myeloproliferative neoplasm: A review of clinical criteria and treatment. Netherlands J Med 70: 159-167.

4. Mi-Ae J, Chul WC (2020) Recent insights regarding the molecular basis of myeloproliferative neoplasm. Korean $\mathrm{J}$ Intern Med 35: 1-11.

5. Macdonald D, Cross N (2027) Chronic myeloproliferative disorders: The role of tyrosine kinases in pathogenesis, diagnosis and therapy. Pathobiology 74: 81-88.

6. Tefferi A, Gilliland GD (2007) Oncogenes in myeloproliferative disorders. Cell Cycle 6: 550-566.

7. Grinfeld J, Nangalia J, Green AR (2017) Molecular determinants of pathogenesis and clinical phenotype in myelopro- liferative neoplasms. Haematologica 102: 7-17.

8. Pandey R, Kapur R (2015) Targeting phosphatidylinositol-3-kinase pathway for the treatment of philadelphia-negative myeloproliferative neoplasms. Molecular Cancers 14: 1-16.

9. Martinelli G, Soverini S, Rosti G, Cilloni D, Baccarani M (2005) New tyrosine kinase inhibitors in chronic myeloid leukemia. Haematologica 90: 534-541.

10. Peiris MN, Li F, Donoghue DJ (2019) BCR: A promiscuous fusion partner in hematopoietic disosrders. Oncotargets 10: 2738-2754.

11. Walz C, Sattler M (2006) Novel targeted therapies to overcome Imatinib Mesylate resistance in chronic myeloid leukemia (CML). Crit Rev Oncol Hematol 57: 145-164.

12. Bing L, Raajit R, Zhijian X (2019) Targeted therapies for myeloproliferative neoplasms. Biomark Res 7: 15.

13. Shannon EE, Ann M (2017) Targeting specific mutations in myeloproliferative neoplasms. Eur Hematol Association 11: 156-159.

14. Nahla H (2018) JAK 2 and its regulators beyond myeloproliferative neoplasms. J Tumor Med Prev 2: 555598.

15. Leroy E, Constantinescu SN (2017) Rethinking JAK 2 inhibition towards novel strategies of more specific and versatile janus kinase inhibition. Leukemia 31: 1023-1038.

16. Shank K, Andrew D, Priya K, Maria K, Julie TF, et al. (2020) Mathematical modeling reveals alternative JAK inhibition treatment in myeloproliferative neoplasms. Haematologica 105: e91-e94.

17. Gorre ME, Mohammed M, Ellwood K, Hsu N, Paquette R, et al. (2001) Clinical resistance to STI-571 cancer therapy caused by BCR-ABL gene mutation or amplification. Science 293: 876-880.

18. Engelman JA, Zejnullahu K, Mitsudomi T, Song Y, Hyland C, et al. (2007) MET amplification leads to gefitinib resistance in lung cancer by activating ERBBS signaling. Science 316: 1039-1043.

19. Verstovsck S, Kantarjian H, Mesa RA, Pardanani AD, Cortes-Franco J, et al. (2010) Safety and efficacy of INCB018424, a JAK1 and JAK2 inhibitor in myelofibrosis. New Engl J Med 363: 1117-1127.

20. Azam P, Nafiseh S, Adib S, Micheal D, Sorin D (2018) A novel computational approach for drug repurposing using system biology. Bioinformatics 34: 2817-2825.

21. Ogbuabor AO, Achukwu PU, Ufelle SA, Ogbuabor DC, Nebo EE (2019) The cytokine network: An integrated approach to understanding the mechanism of unexplained recurrent implantation failures. J Cytokine Biol 4: 130.

22. Amit KB (2015) Computation in analyzing inflammation: $A$ general perspective. Interdisp J Microinflammation 2: 1-4.

23. Aikaterini K, Elisavet CV (2018) Diagnostic molecular techniques in hematology: Recent advances. Ann Transl Med 6: 242.

24. Casie C, Holyoake T (2017) Preclinical approaches in chronic myeloid leukemia: From cells to system. Experimental Hematol 47: 13-23.

25. Kreutz C, Jens T (2009) System biology: Experimental design. FEBS J 276: 923-942.

26. Ashyraliyev M, Yves FN, Jaap AK, Joke GB (2009) System biology: Parameter estimation for biochemical models. FEBS J 276: 886-902.

27. Benavente L, Goldberg A, Henrietta M, Joseph C, Micheal $Z$, et al. (2015) The application of system biology to biomanufacturing. Pharm Bioprocess 3: 341-355. 